\title{
Diagnostic Evaluation of Insulinoma: A Case Report
}

\author{
Maximilian Pinho Schwermann*1, Matheus de Souza Mendes ${ }^{1}$, André Nunes Benevides ${ }^{2}$, Francisco Julimar \\ Correiade Menezes ${ }^{3}$ and José Eudes Bastos Pinho ${ }^{4}$
}

${ }^{1}$ Academic of Medicine of the University of Fortaleza, Brazil

${ }^{2}$ Resident of Digestive Surgery of the General Hospital of Fortaleza, Brazil

${ }^{3}$ Medical Surgeon and Professor of the University of Fortaleza, Brazil

${ }^{4}$ Coordinator of Digestive Surgery of the General Hospital of Fortaleza, Brazil

Received: 䱁: December 05, 2018; Published: 制: December 14, 2018

*Corresponding author: Maximilian Pinho Schwermann, Academic of Medicine of the University of Fortaleza, Brazil

\begin{abstract}
Insulinoma is the most common pancreatic neuroendocrine tumor. The clinical manifestation is diverse, some of which may cause irreversible neurological deficits due to persistent hypoglycemia. This article reports a case of a 17-year-old patient who presented with visual scotoma, somnolence and loss of consciousness with blood glucose documentation of $15 \mathrm{mg} / \mathrm{dL}$. He was admitted and underwent imaging tests. Tomography and Magnetic Resonance imaging did not clearly identify the pancreatic lesion, but tumor lesions were identified after arterial stimulation with calcium. Patient was submitted to surgical enucleation by laparotomy. In this context, there is still much discussion about the optimal sequence of exams for the exact tumor location. However, the use of less invasive exams such as arterial calcium stimulation is essential for adequate surgical resection.
\end{abstract}

\section{Introduction}

Insulinoma is the most common pancreatic-producing neuroendocrine tumor, with an estimated incidence of $0.4 \%$ in the population and accounts for $70 \%$ to $75 \%$ of the causes of hyperinsulinemia. Most insulinomas originate sporadically (90\%), about $5 \%$ to $10 \%$ of cases are associated with hereditary syndromes, such as Multiple Endocrine Neoplasia type 1 (MEN1) and less than $10 \%$ of lesions are malignant [1,2]. Lesions generally occur throughout the pancreas, with a slight predominance of head location [3]. Up to $90 \%$ of the lesions are smaller than 2 $\mathrm{cm}$ and larger lesions, as well as malignant lesions, tend to have more severe symptoms [4]. In 1938, Whipple defined the classical manifestations of patients with insulinoma. Whipple's triad consists of: neuroglycopenic symptoms, resolution of symptoms with glucose administration and serum glucose levels $<40 \mathrm{mg}$ $/ \mathrm{dl}$ and is present in up to $90 \%$ of patients [5]. The duration of symptoms until diagnosis varies from some months and even years. Several laboratory methods and diagnostic imaging methods have been suggested for an early evaluation in suspected patients.

In this context, this intense discussion of diagnostic methods has been supported by the fact that tumor localization is extremely

important for evaluation and surgical programming [6]. Although most of the lesions can be identified by Computed Tomography (CT), other diagnostic methods have gained importance, such as somatostatin analogue guided scintigraphy and arterial calcium stimulation [7]. From a clinical case with classic symptomatology, but with difficulty locating the tumor, we reviewed the literature and described a flowchart for preoperative diagnostic propaedeutics for insulinoma localization.

\section{Case Report}

A 17-year-old male, presented in February 2017 an episode of visual scotoma, drowsiness and loss of consciousness, and he was sent for medical care. At this time, capillary glycemia dosage revealed a serum level of $15 \mathrm{mg} / \mathrm{dl}$. Intravenous glucose was administered, and the patient returned home asymptomatic. Thereafter, the patient presented several similar episodes, usually in the morning, upon waking and they improved after eating sweet foods. Patient denied abdominal pain, nausea, vomiting or seizures. He also denied weight loss and changes in bowel habits. After several episodes, the patient was referred for tertiary evaluation at the General Hospital of Fortaleza. He initiated a long-term fasting 
test (72 hours), with glycemia of $37 \mathrm{mg} / \mathrm{dl}$, insulin of $11.8 \mathrm{U} / \mathrm{ml}$, pro-insulin of $1.53 \mathrm{pmol} / \mathrm{L}$ and peptide C of $2.88 \mathrm{ng} / \mathrm{ml}$. Such findings suggested endogenous hyperinsulinemia hypoglycemia. He performed a (CT) scan of the abdomen and pelvis that showed no structural alterations in the pancreatic parenchyma.

He performed nuclear magnetic resonance imaging of the abdomen (MRI) that failed to clearly identify lesion in the pancreatic parenchyma. The same thing happened with endoscopic ultrasonography. He then performed catheterization with calcium stimulus, identifying insulin peak after stimulation in the gastroduodenal artery, thus identifying pancreatic head injury (Table 1). Patient underwent surgical enucleation by laparotomy, with palpation of nodular lesion, well delimited, measuring $1.2 \mathrm{x}$ $1.0 \mathrm{~cm}$, in the posterolateral face located in the pancreatic cool-head transition. No other lesions were identified. In the postoperative period, the patient evolved without pancreatic fistula, without new episodes of hypoglycemia and without the need for parenteral glucose support and was discharged for outpatient follow-up.

Table 1: Dosage of insulin in hepatic veins after selective intraarterial stimulation with calcium gluconate.

\begin{tabular}{|c|c|c|c|}
\hline Time & $\begin{array}{c}\text { Splenic } \\
\text { artery }\end{array}$ & $\begin{array}{c}\text { Gastroduodenal } \\
\text { artery }\end{array}$ & $\begin{array}{c}\text { Superior } \\
\text { mesenteric } \\
\text { artery }\end{array}$ \\
\hline Baseline & 46,5 & 48,3 & 47,4 \\
\hline 30 seconds & 42,9 & 245 & 44,3 \\
\hline 60 seconds & 50,6 & $>300$ & 43,1 \\
\hline 120 seconds & 71,6 & 297 & 46,3 \\
\hline \multicolumn{3}{|c|}{ Peripheral insulin: 28,1} \\
\hline
\end{tabular}

\section{Discussion}

Insulinoma accounts for about $60 \%$ of pancreatic islet-related neoplasms. The tumor is characterized by small dimensions $(0.8$ to $1.5 \mathrm{~cm}$ ), well encapsulated and vascularized. Early diagnosis is critical, as late sequelae include irreversible neurological symptoms. Therefore, an intensive investigation is justified both by invasive and non-invasive methods in order to properly identify the lesion. There is also discussion about how invasive this research should be, and what is the ideal sequence of tests to be performed, given the range of options currently available. Since its initial description in 1930 by Roscoe Graham [8], pancreatic preservation surgery (tumor enucleation) has been indicated as a definitive therapeutic option, since this technique is associated with lower rates of Diabetes Mellitus in the postoperative period $[9,10]$. Clinical and laboratory diagnosis of insulinoma should meet certain criteria: symptoms of hypoglycaemia, documentation of glucose levels below $40 \mathrm{mg} / \mathrm{dL}$, elevated insulin and C-peptide levels and absence of plasma sulfonylurea. The laboratory gold standard is the 72-hour prolonged fasting test, which is used to monitor endogenous insulin suppression against hypoglycemia, as well as to observe symptoms, blood glucose levels, and other laboratory tests such as C-peptide levels, insulin, proinsulin, sulfonylurea and beta-hydroxybutyrate [9].

To identify the pancreatic tumor, several imaging tests have been used to guide surgical therapy. The exam of choice is abdominal ultrasonography for various abdominal conditions. However, for the evaluation of insulinomas, there is no high sensitivity (86.5\%). The examination does not usually identify tumors smaller than $2 \mathrm{~cm}$ and has limitations in visualizing lesions in the caudal portion of the organ. Intraoperative ultrasonography is extremely useful in guiding tumor dissection processes and evaluating its relationship with intraparenchymal structures (main pancreatic duct), and it is a method used when direct palpation of the organ does not identify the lesion. Computed tomography with contrast, as it is not an invasive exam and has a high sensitivity for the disease (95.3\%), is usually a method used for initial investigation and is the standard exam for preoperative evaluation of insulinomas. Insulinoma is hyper vascularized and, therefore, is best visualized in an early arterial phase, being possible to identify lesions smaller than $1.5 \mathrm{~cm}$. Magnetic resonance imaging has also been suggested as an auxiliary examination in the identification of lesions, with a higher sensitivity than CT $(98.7 \%)[9,11]$. Even in the face of these characteristics, these tests may fail to detect tumors smaller than $1 \mathrm{~cm}$ but assist in the evaluation of disease metastases or in the identification of large malignancies.

Invasive options gain importance in the identification of small lesions that have not been visualized in previous methods, such as endoscopic ultrasonography and selective intra-arterial calcium stimulation. The first one is used by several centers as the first option due to its high capacity to identify lesions smaller than 5 $\mathrm{mm}$. However, it is noteworthy that endoscopic ultrasonography presents low sensitivity for lesions that are located beyond the head of the pancreas. This method is extremely useful when preoperative biopsies are required. Selective intra-arterial calcium stimulation is based on the ability of the tumor to secrete insulin against a calcium stimulus. It is performed through venous catheterization of the hepatic veins and the samples of insulin are collected after calcium stimulation and arterial catheterization in the gastroduodenal, superior mesenteric and splenic arteries, where the infusion of calcium is performed. The collections are performed at 30,60, 90 and 120 seconds after stimulation. Currently, the use of this examination is considered if the tumor cannot be located with two non-invasive imaging tests (Figure 1). Even if this modality does not directly establish the precise location of the tumor in individuals who do not present arterial anatomical variations, this examination becomes an extremely useful artifice for adequate surgical programming.

The use of scintigraphy with somatostatin analogs for tumor localization has been employed for several pancreatic neuroendocrine tumors. However, in addition to being a high-cost examination and still not widely available, it has an insulinomas identification rate of $47 \%$. Non-expression of somatostatin receptors by some insulinomas has limited their use for this condition $[9,11,12]$. Tumor enucleation is the main technique used for benign and superficial tumors. In addition to being the most performed procedure for insulinomas, it is the one with the lowest rates of postoperative complications. For tumors greater than $2.5 \mathrm{~cm}$ or $2 \mathrm{~mm}$ from the main pancreatic duct, enucleation is associated with elevated levels of pancreatic fistulas, and should be considered segmental resections for curative treatment purposes, 
either with duodeno pancreatectomy or with distal pancreatectomy [11]. Lesions with malignant characteristics should be treated with segmental resection associated with lymphadenectomy [12]. Both laparoscopic and the laparotomic approach are viable options for the treatment of insulinomas.

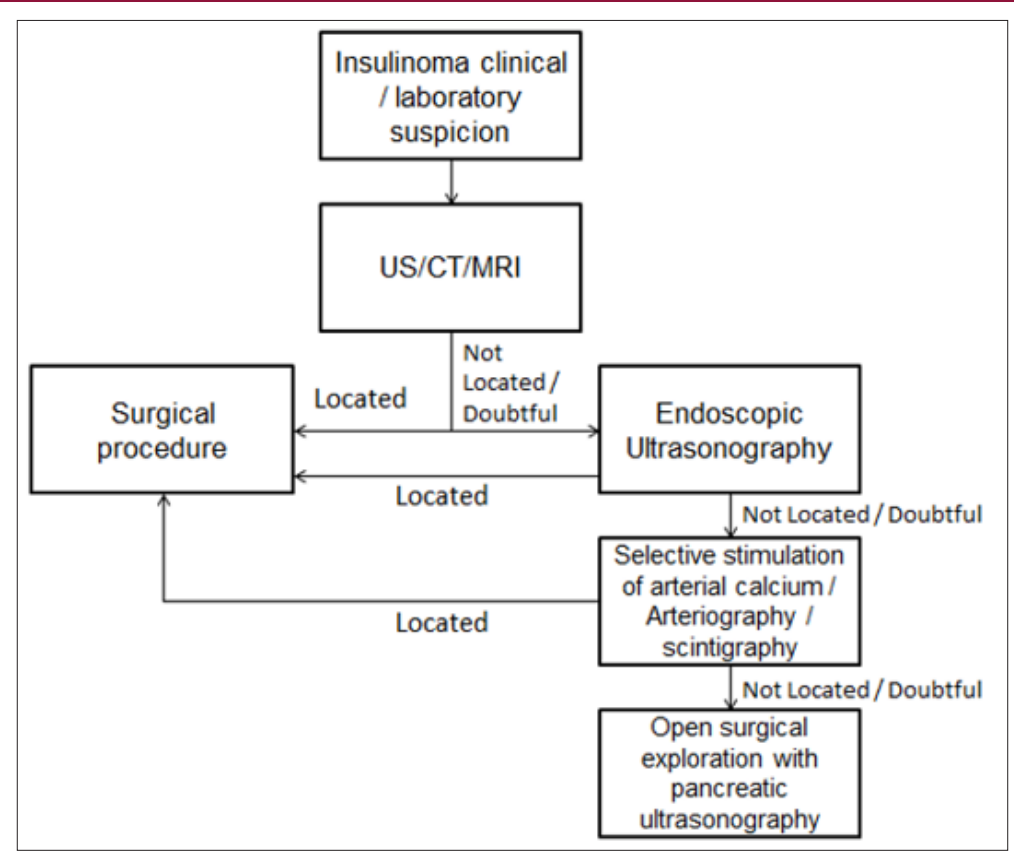

Figure 1: Crown height measurement via digital caliper.

Laparoscopic enucleation has been recommended for tumors located in the body and tail of the pancreas and whose location was easily defined in the preoperative period, through imaging tests. Deep lesions in the pancreatic parenchyma, with difficulty of identification in the preoperative period, located in the head or dorsal region and that may require intraoperative ultrasonography, should be resected through the open route [11].

Clinical suspicion of insulinoma should be thoroughly investigated to identify the lesion. Early treatment of these tumors is related to high cure rates and prevents the irreversible neurological changes that may arise.

\section{Conclusion}

The present report demonstrates the difficulty of several methods in identifying the precise location of insulinomas. The ideal sequence of preoperative exams to establish the best proper surgical management is still under discussion. However, the sequential use of less invasive exams for options such as arterial calcium stimulation is essential for surgical programming. In this context, enucleation is effective even for non-localized tumors precisely in the preoperative period and is an option for tumors with no malignant characteristics localized to the pancreatic surface.

\section{References}

1. Aggeli C, Nixon AM, Karoumpalis I, Kaltsas G, Zografos GN, et al (2016) Laparoscopic surgery for pancreatic insulinomas: an update. Hormones (Athens)15(2):157-169.
2. Öberg K (2018) Management of functional neuroendocrine tumors of the pancreas. Gland surgery 7(1): 20-27.

3. Brown E, Watkin D, Evans J, Yip V, Cuthbertson DJ et al. (2018) Multidisciplinary management of refractory insulinomas. Clin Endocrinol (Oxf) 88(5): 615-624.

4. Baudin E, Caron P, Lombard-Bohas C, Tabarin A, Mitry E et al. (2013) Malignant insulinoma: recommendations for characterisation and treatment. Ann Endocrinol 74: 523-533.

5. Sheeva KP, Douglas GA (2016) Pancreatic neuroendocrine tumors: contemporary diagnosis and management. Hospital Practice 44(3):109119.

6. Tamm EP, Bhosale P, Lee JH, Rohren EM 2016 State-of-the-art imaging of pancreatic neuroendocrine tumors. Surg Oncol Clin N Am 25(2): 375400 .

7. Sharma P, Arora S, Dhull VS, Naswa N, Kumar R, et al (2015) Evaluation of (68) Ga-DOTANOC PET/CT imaging in a large exclusive population of pancreatic neuroendocrine tumors. Abdom Imaging 40(2): 299-309.

8. Medina ZP, Lucio FJ (2016) Insulinoma: revisión actual de manejo y tratamiento. Revista Médica MD 7(2): 84-91.

9. Michele O, Angela P, Jole V, Diana A, Laterza MM et al. (2016) Pancreatic neuroendocrine tumors: nosography, management and treatment. International Journal of Surgery 28:156-162.

10. Anderson, Carinne W, Joseph JB (2016) Clinical presentation and diagnosis of pancreatic neuroendocrine tumors. Surgical Oncology Clinics 25(2): 363-374.

11. Mehrabi A, Fischer L, Hafezi M, Dirlewanger A, Grenacher L et al. (2014) A systematic review of localization, surgical treatment options, and outcome of insulinoma. Pancreas 43(5): 675-686.

12. Amareshwar C, David AK (2016) Surgical management of pancreatic neuroendocrine tumors. Surgical Oncology Clinics 25(2): 401-421. 
ISSN: 2574-1241

DOI: $10.26717 / B J S T R .2018 .12 .002209$

Maximilian Pinho Schwermann. Biomed J Sci \& Tech Res

This work is licensed under Creative

Commons Attribution 4.0 License

Submission Link: https://biomedres.us/submit-manuscript.php

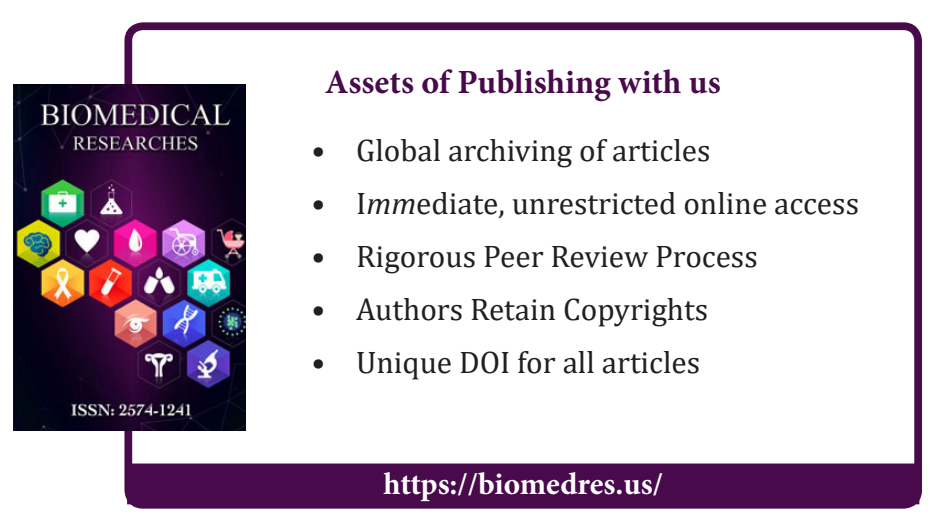

